\title{
Computational insights into binding mechanism of drugs as potential inhibitors against SARS-CoV-2 targets
}

\author{
Mahreen Arooj $^{1}$ (D) $\cdot$ Ihsan Shehadi $^{1}$ (D) Chahlaa N. Nassab ${ }^{1}$ (D) $\cdot$ Ahmed A. Mohamed $^{1}$ (D)
}

Received: 4 July 2021 / Accepted: 27 August 2021 / Published online: 30 August 2021

(C) Institute of Chemistry, Slovak Academy of Sciences 2021

\begin{abstract}
Because of the scale of the novel coronavirus (COVID-19) pandemic and the swift transmission of this highly contagious respiratory virus, repurposing existing drugs has become an urgent treatment approach. The objective of our study is to unravel the binding mechanism of the Food and Drug Administration (FDA)-approved dexamethasone (Dex) and boceprevir (Boc) drugs with selected COVID-19 protein targets SARS-CoV-2 spike protein C-terminal domain (spike-CTD), main protease $\left(\mathrm{M}^{\mathrm{pro}}\right)$, and interleukin-6 (IL-6). Another objective is to analyze the effects of binding Dex and Boc drugs on the interactions of viral spike protein to human angiotensin-converting enzyme 2 (hACE2). Molecular docking and one-microsecond-long molecular dynamics simulations of each of the six protein-drug complexes along with steered molecular dynamics (SMD) and umbrella sampling (US) methods have revealed the binding mode interactions and the physicochemical stability of the three targeted proteins with two drugs. Results have shown that both drugs bind strongly with the three protein targets through hydrogen bonding and hydrophobic interactions. A major finding from this study is how the binding of the drugs with viral spike protein affects its interactions at the binding interface with hACE2 protein. Simulations of drug-bound spike-CTD with hACE2 show that due to the presence of a drug at the binding interface of spike-CTD, hACE2 is being blocked from making putative interactions with viral protein at such interface. These important findings regarding the binding affinity and stability of the two FDA-approved drugs with the main targets of COVID-19 along with the effect of drugs on hACE2 interactions would contribute to COVID-19 drug discovery and development.
\end{abstract}

Keywords Dexamethasone $\cdot$ Boceprevir $\cdot$ Stability $\cdot$ Spike protein $\cdot$ hACE2 $\cdot$ Molecular dynamics calculations

\section{Introduction}

The novel human severe acute respiratory syndrome coronavirus SARS-CoV-2 that appeared in late 2019 has been spreading rapidly all over the globe (Castagnoli et al. 2020).

Mahreen Arooj and Ihsan Shehadi contributed equally to this manuscript.

Mahreen Arooj

marooj@sharjah.ac.ae

$\triangle$ Ahmed A. Mohamed

ah.mohamed@sharjah.ac.ae

Ihsan Shehadi

ishehadi@sharjah.ac.ae

Chahlaa N. Nassab

u19103696@sharjah.ac.ae

1 Department of Chemistry, College of Sciences, University of Sharjah, Sharjah 27272, UAE
Physical distancing and personal protective equipment have been the most effective practices against the transfer of COVID-19 (Chandrasekharan et al. 2020; Sud 2020). Due to the unavoidable course of actions to stimulate the global economy, the rush for therapeutic drugs and vaccines has become a target for basic and applied research (Jackson et al. 2020). Researchers around the globe have been trying to fathom SARS-CoV-2 and explore its pathophysiology (Gurwitz 2020). During these pursuits, repositioning of the FDA-approved drugs is considered the most pivotal route (Jackson et al. 2020; Gurwitz 2020; Elfiky 2020). In the current study, two FDA-approved dexamethasone (Dex) and boceprevir (Boc) drugs are selected due to their contemporary role in the treatment of COVID-19 (Johnson and Vinetz 2020; Fu et al. 2020) (Fig. 1).

Dex is a corticosteroid drug that is used for the treatment of immunosuppressant and anti-inflammatory conditions. Boc drug is clinically a protease inhibitor which was approved by the FDA in 2011 for the treatment of 
Fig. 1 Three-dimensional structures of dexamethasone (Dex) and boceprevir (Boc) drugs are represented in stick-style and color-coded by element $(\mathrm{N}$ : blue, O: red, H: white), and only carbon atoms are in yellow and green, respectively. Nonpolar hydrogens are removed for clarity
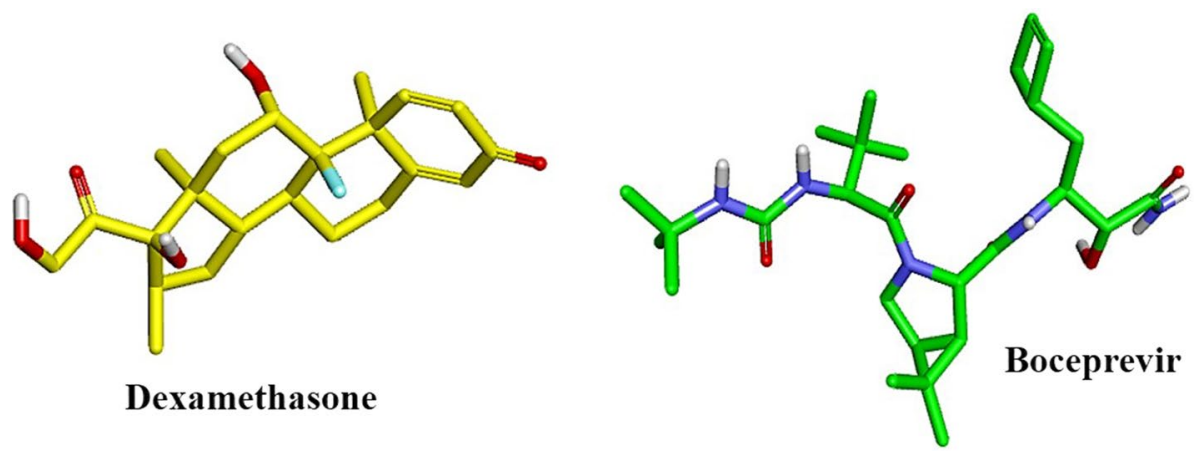

chronic hepatitis $\mathrm{C}$ virus ( $\mathrm{HCV}$ ). In this research, the binding mechanism of Dex and Boc drugs with three important COVID-19 protein targets including main protease ( $\mathrm{M}^{\text {pro }}$, also called 3CLpro), SARS-CoV-2 spike protein C-terminal domain (spike-CTD), and interleukin-6 (IL-6) was investigated using various computational techniques. Recently, few computational studies are reported involving these drugs and proteins (Khan and Htar 2020; Nayeem et al. 2021, Jani et al. 2021). However, this is the first report on the binding mechanism of the selected drugs with the selected protein targets of COVID-19. It is very crucial to understand the binding mechanism of Dex due to its important role in COVID-19 treatment (Johnson and Vinetz 2020). Researchers in the UK used Dex in the national COVID-19 clinical trials and found it to be effective for the treatment of critically ill patients (Ullrich and Nitsche 2020). Findings from the trials showed that the treatment with Dex had reduced the mortality rate by one-third for patients on ventilators. In addition, the mortality rate was reduced by one-fifth in patients who necessitated oxygen only (Ullrich and Nitsche 2020). A preliminary report on the use of Dex in hospitalized patients with Covid-19 showed impressive results with a reduced mortality rate in patients (RECOVERY Collaborative Group. 2020). Another clinical trial that included 299 patients reported that the number of days alive and free from mechanical ventilation was considerably greater for patients treated with Dex with standard care as compared with standard care alone (Ullrich and Nitsche 2020). Another very recent review regarding the therapeutic potential and risks of Dex for COVID-19 patients also suggested that drug repositioning screening of dexamethasone may contribute to the COVID-19 treatment efficiently for critically ill patients based on risk-benefit ratio monitoring (Ullrich and Nitsche 2020). World Health Organization (WHO) also issued a guideline on the use of Dex and other corticosteroids for the treatment of patients with severe and critical COVID-19 (Ullrich and Nitsche 2020). Another selected drug in this study is Boc which had been proven to be effective in the treatment of COVID-19 and had been supported by recent preclinical studies (Ma et al. 2020; Fu et al. 2020). Results of the preclinical studies had also shown that Boc effectively inhibited SARS-CoV-2 in Vero cells by targeting coronavirus main protease ( $\mathrm{M}^{\text {pro }}$, also called $3 \mathrm{CL}$ pro). $\mathrm{M}^{\text {pro }}$ is a vital target for drug development against COVID-19 due to its central role in the life cycle of coronavirus (Ullrich and Nitsche 2020).

SARS-CoV-2 spike protein is a vital COVID-19 target that binds to the human angiotensin-converting enzyme (hACE-2) expressed on the alveolar epithelial cells in the lungs (Gavor et al. 2020) (Fig. 2). Due to the binding of the viral spike protein to the hACE-2 receptor, the virus can successfully enter the host cells leading to endocytosis of the lung alveolar epithelial cells which causes irreversible pneumocytes damage to lung tissues (Hasan et al. 2020). Targeting receptors to mitigate pulmonary inflammation in COVID-19 patients is also an imperative approach (Mehta et al. 2020). SARS-CoV-2 often results in mild or highly acute respiratory syndrome with the release of proinflammatory cytokines, including interleukin-6 (IL-6) (Ho et al. 2015). Inhibition of IL-6 activity may attenuate pulmonary inflammation in COVID-19 patients (Della-Torre et al. 2020).

Computational methods are very significant to answer fundamental biologically important questions related to ligand-protein binding affinity, complex stability, and binding mechanisms (Bentel et al. 2017; Harb et al. 2017; Iqbal et al. 2019; Shehadi et al. 2020; Wang et al. 2007; Wei et al. 2006; Arooj et al. 2020). They have also been used extensively to identify the potential inhibitors of SARS-CoV-2 (Wang et al. 2020; Ciliberto and Cardone 2020; Rahman et al. 2020). However, none of these studies evaluated the effect of the binding mode interactions of Dex with viral spike proteins. Moreover, the binding mode information of Boc drug with putative targets of COVID-19 including viral spike proteins along with IL-6 receptor has not been reported yet.

The significant biological question that requires an urgent response is the effect of drug binding on the formation of spike-CTD complexed with hACE2. Can binding Dex and Boc drugs with CTD of SARS-CoV-2 spike protein weaken their interactions with hACE2? To the best of our knowledge, no such study has been conducted to explore the 


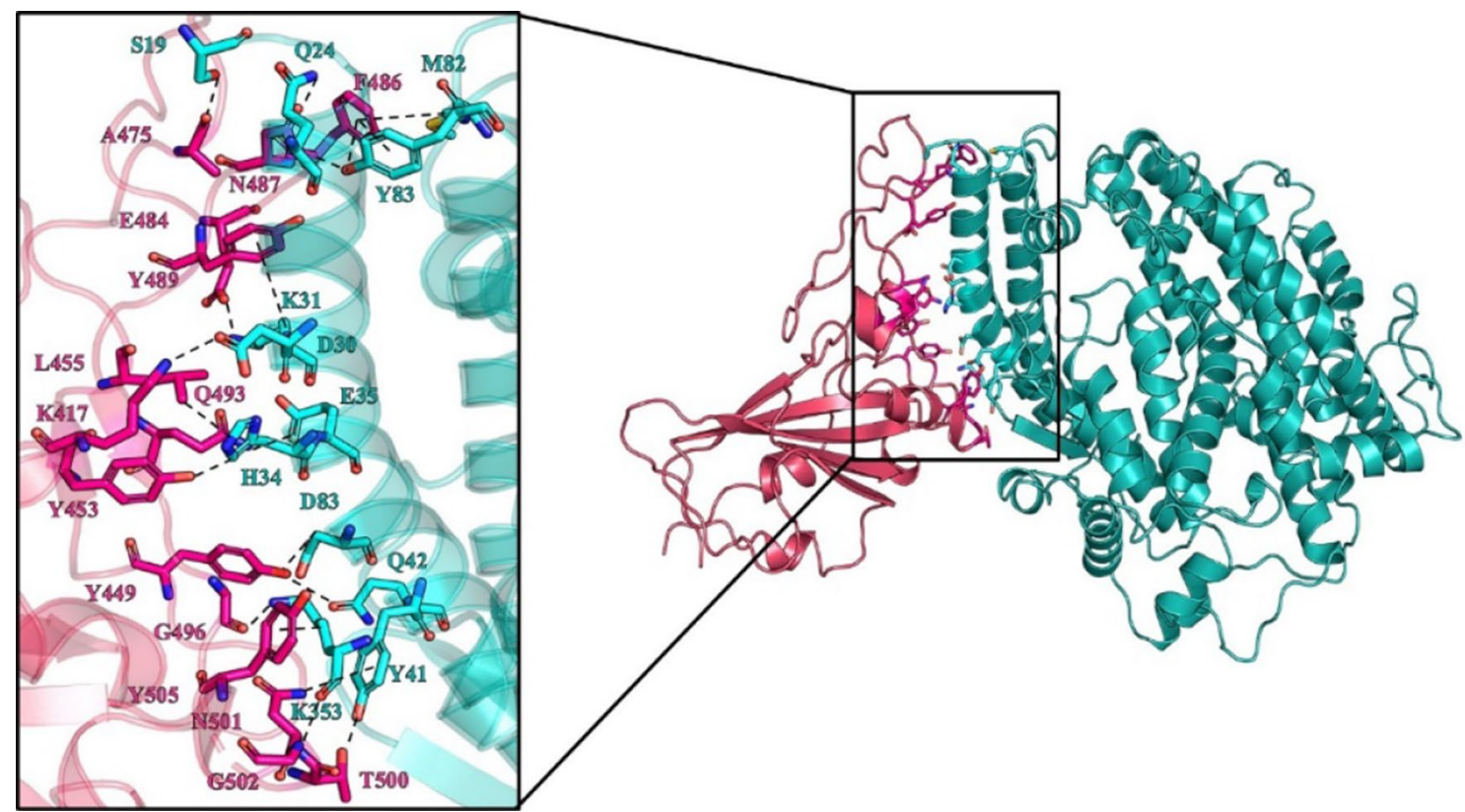

Fig. 2 Co-crystallized complex structure of SARS-CoV-2 spike protein C-terminal domain (spike-CTD) with human ACE2. Structures of spike-CTD and hACE2 are represented in red and green ribbons,

effects of binding of Dex and Boc drugs with SARS-CoV-2 spike protein on the interactions with hACE2. To answer these important queries, the current study aims to investigate the binding mechanism of Dex and Boc drugs with the essential targets of SARS-CoV-2 using diverse computational tools including molecular docking and molecular dynamics (MD) simulations methods. The novelty of our study also lies in providing insights into the binding mode of SARS-CoV-2 spike protein with hACE2 in the presence of a drug bound with CTD of the viral spike protein. Findings from this research work are critical in understanding the interactions and development of drugs as potential inhibitors with key targets of COVID-19.

\section{Computational methods}

\section{Structure preparation of proteins and drugs}

Three-dimensional (3D) crystal structure of SARS-CoV-2 spike receptor-binding domain complexed with hACE-2 in PDB ID 6LZG was downloaded from Protein Data Bank (PDB) with a resolution of $2.50 \AA$ (Wang et al. 2020). SARS-CoV-2 main protease $\left(\mathrm{M}^{\mathrm{pro}}\right)$ complexed with Boc (PDB ID: 7K40, resolution of $1.35 \AA$ ) and human IL-6 (PDB ID: $1 \mathrm{ALU}$, resolution of $1.90 \AA$ ) $3 \mathrm{D}$ crystal structures were obtained from Protein Data Bank (Somers et al. 1997). Water molecules and heteroatoms were removed from all proteins using Discovery Studio (DS) client 2020 respectively. Residues involved in the interactions at the interface of both proteins are displayed in stick style. Nonpolar hydrogens are hidden for clarity. Interactions are indicated in dashed lines

program, and proteins were prepared using Prepare Protein tool implemented in DS. The final protein structures were saved as PDBQT in AutoDock 4.2 (Trott and Olson 2009). 3D structures of Dex and Boc drugs were retrieved from the PubChem website and were prepared using Prepare ligand tool in DS.

\section{Preparation of protein-drug complexes}

A molecular docking technique was employed to prepare protein-drug complexes. A preliminary molecular docking validation calculation was performed by separating the crystal structure-bound Boc drug and redocking using AutoDock 4.2 (Goodsell et al. 1996). The binding mode predicted by docking was compared to the co-crystallized structure of $\mathrm{M}^{\text {pro }}$ with Boc. Results confirmed that docking reproduced the co-crystallized binding mode of Boc at the active site of $\mathrm{M}^{\text {pro }}$ (Fig. S1).

After the validation, docking was performed to prepare protein-drug complexes. Lamarckian genetic algorithm (LGA) was used for the conformational search and protein-drug interactions investigation. The following search girds were employed: $(-34.33,26.02,8.03)$ size of $25 \AA$ for spike protein, $(10.14,-25.26,-23.45)$ size of $30 \AA$ for $\mathrm{M}^{\text {pro }}$ protein, and $(0.55,-15.67,0.28)$ size of $25 \AA$ for IL-6 as shown in Fig S2. Energy evaluations of 250,000 were completed to obtain the minimum free energy of protein-drug complexes. $\Delta G^{\text {Autodockbind }}$ scoring function was used for the energy calculations which consider the 
following energy terms: van der Waals $\left(\Delta H_{v d W}\right)$, hydrogen bonding $\left(\Delta H_{h \text {-bond }}\right)$, electrostatic $\left(\Delta H_{\text {elec }}\right)$, desolvation free energy $\left(\Delta H_{\text {desolv }}\right)$, and conformational entropy $\left(\Delta S_{\text {conf }}\right)$.

$\Delta G^{\text {Autodockbind }}=\Delta H_{v d W}+\Delta H_{h-\text { bond }}+\Delta H_{\text {elec }}+\Delta H_{\text {desolv }}+\Delta S_{\text {conf }}$

A total of 9 conformations of each protein-drug complex were obtained from the docking calculations. Conformations of protein-drug complexes were analyzed based on their binding energies. The cutoff value of 2.0 $\AA$ RMSD was used for clustering analysis as implemented in AutoDock 4.0. After performing a detailed binding mode analysis of each conformation using DS, the lowest energy conformation for each of the complexes was selected for molecular dynamics simulations, taking into consideration the type and strength of interactions.

\section{Molecular dynamics simulations}

Following the protein-drug complexes preparation via molecular docking, MD simulations were performed to examine the stability of the generated systems and further confirm the binding mechanisms. MD simulations were performed with GROMACS 2019.2 software package (Abraham et al. 2015) using the OPLS-AA force field. Total six simulations were run for the three proteins and the two drugs complexes. The OPLS-AA force field parameters of the two drugs were generated using the web server LigParGen (Dodda et al. 2017). Each protein-drug complex was subjected to periodic boundary conditions (PBCs) in a cubic box solvated using the TIP4P water model. Counter ions were added to neutralize the systems as required. The simulations were conducted with the NPT and Particle-Mesh Ewald (PME) method using a cutoff of $1.2 \mathrm{~nm}$. The electrostatic potential calculations with PBCs were employed in these simulations (Darden et al. 1993). Along with the PME interpolation order of 4, the Fourier spacing with $0.12 \mathrm{~nm}$ was applied. SHAKE algorithm was applied to constrain the H-bonds. All six protein-ligand complexes were subjected to 20,000 iterations for the minimization process using the steepest descent algorithm. Moreover, after minimization, each system was equilibrated for $2 \mathrm{~ns}$ with a time step of $1.0 \mathrm{fs}$. Finally, a production run of $1000 \mathrm{~ns}(1 \mu \mathrm{s})$ was carried out for each of the protein-drug complexes; thus, six microseconds simulations were performed for six protein-drug complexes. The frames from each of the microsecond-long simulations were saved as separate trajectory after every $10 \mathrm{ps}$ in order to analyze each simulated system independently.

Along with these six simulations of six microseconds, two more simulations were conducted to reveal the effect of drug binding on spike-CTD and hACE2 interactions. To study the ability of the drugs to affect spike-hACE-2 interactions, drug-bound spike-CTD protein retrieved from the molecular docking calculations and hACE-2 were kept in a water box and were separated with $15 \AA$ (Fig. S3). Then, simulations were run for $100 \mathrm{~ns}$ to evaluate the binding of hACE2 with spike-CTD in the presence of Dex and Boc drugs.

\section{Steered molecular dynamics (SMD) and umbrella sampling (US)}

To calculate the binding affinity of the protein-drug complexes, a combination of two techniques was used: steered molecular dynamics and umbrella sampling. From the end of the MD simulation of each protein-drug complex, based on clustering thus the representative structure of the top cluster was selected to perform steered molecular dynamics using the center of mass pulling. In this technique, the drug is being pulled away from the binding site of the protein by applying a force on the drug, while positional restraints are applied on the protein. The six protein-drug systems were prepared by placing the complex in a water box elongated along the $\mathrm{x}$-axis. The drug was then forced to leave the binding site of the protein with a pulling force of $100(\mathrm{~kJ} / \mathrm{mol}$. $\mathrm{nm}^{2}$ ). Before the simulation, the system was equilibrated for $100 \mathrm{ps}$; then, pulling was performed for $500 \mathrm{ps}$. Hence 500 conformations of each protein-drug system were produced. Each conformation represented a sampling window that held the drug at an increasing distance away from the protein. Afterward, umbrella sampling was performed. To generate sufficient sampling, 90 sampling windows with a $0.02-\mathrm{nm}$ spacing were selected to perform a 10 -ns simulation time. Then weighted histogram analysis (WHAM) method of calculation was used to extract the potential of mean force (PMF) curve from which binding affinity was calculated (Ngo et al. 2019).

\section{Simulations analysis}

MD simulations produced structural data which were extracted to conduct root-mean-square deviation (RMSD), root-mean-square fluctuation (RMSF), the radius of gyration $(\mathrm{Rg})$, and hydrogen bonds using GROMACS built-in tools including g-rms, g-rmsf, g-gyrate, and g-hbond analyses. In addition, cluster analysis with a cutoff of $0.3 \mathrm{~nm}$ was performed using the g-cluster module. Principle component analysis (PCA) was carried out using g-covar on the backbone atoms of the proteins. Furthermore, the first two eigenvectors ( $\mathrm{PC} 1$ and $\mathrm{PC} 2$ ) which were projected using g-analog module were being used to plot the free energy landscape (FEL) analysis. FEL was generated using python script generateFES.py and was plotted using Gnuplot version 5.2. 


\section{Results and discussion}

\section{Dexamethasone and boceprevir binding modes}

Protein-ligand molecular docking technique was implemented to determine the binding mode of Dex and Boc with COVID-19 protein targets (Fig. 3), and the lowest energy conformations were evaluated. The final binding modes of drug-protein complexes were selected based on the key interactions of drugs with the important residues of the binding pocket of the proteins.

\section{Modulation of structure and dynamics of protein- drug complexes}

To explore the internal motions, conformational changes, stability, and interaction mechanisms of SARS-CoV-2 spike, $\mathrm{M}^{\mathrm{pro}}$, and IL-6 proteins with Dex and Boc drugs, the selected docking conformations of all three proteins complexed with both drugs were subjected to atomistic-level MD simulations. Trajectories generated from MD simulations for all six protein-drug complex systems were evaluated thoroughly using various analyses. The binding of small molecules in the active sites of proteins might have induced conformational changes in their globular structures. Measurement of RMSD of proteins backbone is a common practice to examine their structural deviation and stability. RMSD analysis was performed for each protein and the bound drug at the active site for all simulated systems (Fig. 4).

Average RMSD values of $0.29 \mathrm{~nm}$ and $0.31 \mathrm{~nm}$ for the backbone of spike protein with Dex and Boc drugs, respectively, indicated the stability of protein structure in the presence of the drugs (Fig. 4). Initially, the spike complexed with Boc showed some fluctuation, but for the later part of the simulation, it became stable and maintained RMSD of around $0.31 \mathrm{~nm}$ without much variation until one microsecond. Compared to spike protein-drug complexes, the other proteins of $\mathrm{M}^{\text {pro }}$ and IL- 6 complexed with the drugs presented relatively better stability of the simulated systems, which was evident by the relatively lower averaged RMSD values of 0.26 and $0.22 \mathrm{~nm}$ for the Mpro-Dex and Mpro-Boc protein complexes, respectively. Moreover, IL6-bound drug-protein complexes did not show much variation throughout the simulation time with the lowest averaged RMSD values of 0.21 and $0.20 \mathrm{~nm}$ for Dex and Boc-bound protein complexes, respectively.
Fig. 3 Docked conformations of the protein-drug complexes. Protein is shown in both surface and cartoon representation, and the drugs are shown in sphere representation. A spike-CTD-

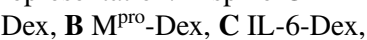
(D) spike-CTD-Boc, $\mathbf{E}$

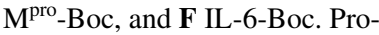
teins are colored in pink, light blue, and orange, respectively. Dex and Boc drugs are colorcoded by element ( $\mathrm{N}$ : blue, $\mathrm{O}$ : red, H: white), and only carbon atoms are in yellow and green, respectively

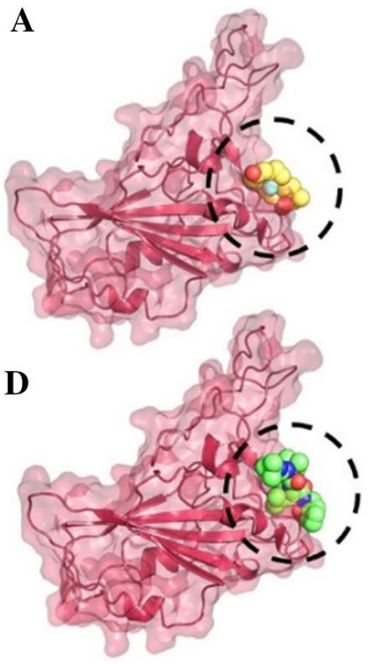

$\mathbf{B}$

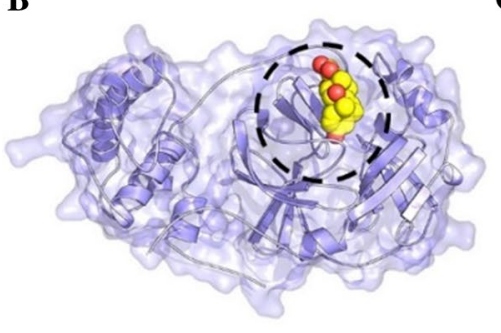

E

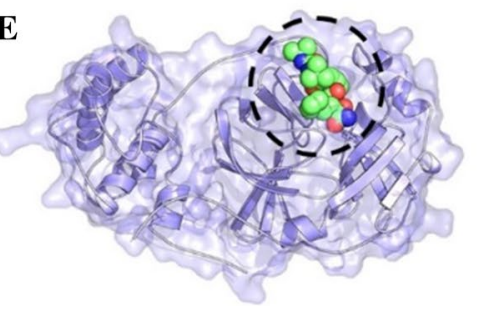

C

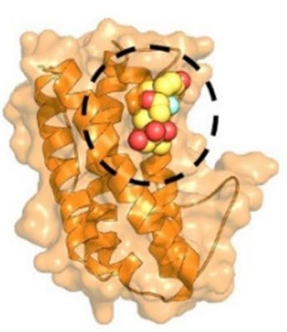

F

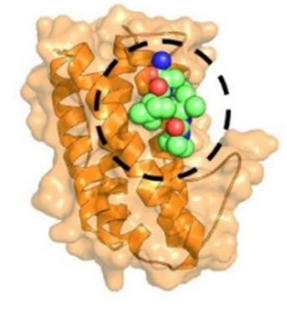

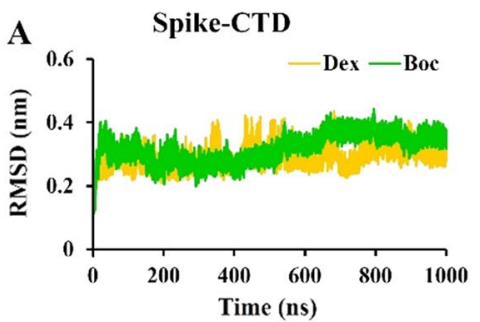
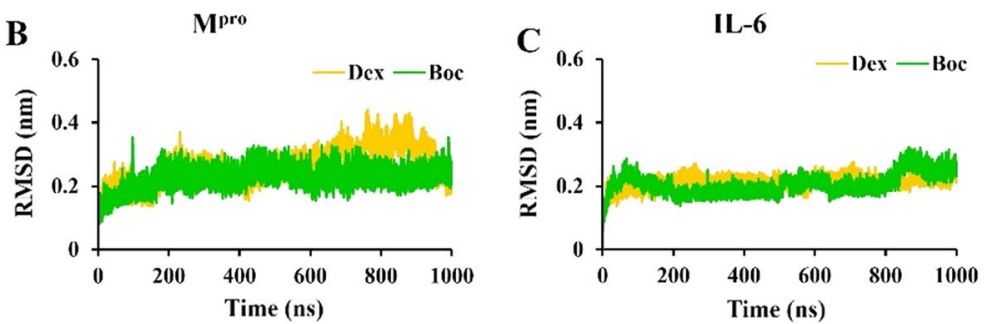

Fig. 4 RMSD graphs of protein backbone for all three proteins. A Spike-CTD, B M ${ }^{\text {pro }}$, C IL-6 were produced from one-microsecond simulations per system 
The radius of gyration $\left(R_{g}\right)$, a parameter that is associated with the structural compactness and overall conformational shape of proteins, was calculated to evaluate the compactness of protein-drug complexes (Fig. S4). Higher values of $R_{g}$ are expected because of the less tight packing of protein structures. The average $R_{g}$ value for spike protein with Dex and Boc drugs was $1.85 \mathrm{~nm}$, while the average $R_{g}$ of the $\mathrm{M}^{\mathrm{pro}}$ protein system with both drugs was the highest with a value of $2.21 \mathrm{~nm}$. Average $R_{g}$ values of IL-6 complexed with Dex and Boc were $1.62 \mathrm{~nm}$ and $1.64 \mathrm{~nm}$, respectively. Overall, the $R_{g}$ plot did not display remarkable changes which indicated that there was no structural deviation in all three proteins.

\section{Flexibility analysis of protein-drug complexes}

To ascertain the local structure and residual flexibility for all six protein-drug complexes, the average fluctuation of the entire protein structure was computed and plotted as RMSF (Fig. 5).

RMSF plots revealed several residual fluctuations at different regions for all three protein structures. The relatively higher peaks were labeled with residue numbers. In the case of spike protein, residues 473-490 and 519-527 belonging to the $\mathrm{N}$ - and $\mathrm{C}$-termini regions with coil conformation exhibited more flexibility compared to the central region of the protein. Various residues in the structure of $\mathrm{M}^{\text {pro }}$ also displayed relatively higher flexibility, especially at
C-terminus. However, all these flexible regions were away from the binding pocket. RMSF of IL- 6 indicated several random fluctuations at regions spanning from $\mathrm{N}$-terminal to C-terminal including 70-78 and 131-135 regions. None of the binding site residues showed much flexibility in all six drug-bound protein systems, thus ascertaining the putative binding modes of both drugs in all three proteins.

\section{Free energy landscapes}

Collective atomic motions are important for proteins to accomplish their specific functions. Principal component analysis (PCA) was performed to determine the conformational fluctuations of all six protein-drug complexes. 3D projections of Gibbs free energy landscapes for the first two principal components (PCA1, PCA2) projected by the $\mathrm{C}^{\alpha}$ atom were plotted to present the tertiary conformations via the populated cluster motions (Fig. 6).

Gibbs free energy landscapes presented the global energy minima states thus are showing the energetically preferred protein configurations in all six protein-drug complexes. The dark blue color in the 3D projection indicated the minimum energy conformations of proteins with maximum stability, whereas energetically unfavorable configurations of proteins were represented in red in the 3D plot. Spike protein systems complexed with both drugs exhibited relatively high Gibbs free energy of $10.95 \mathrm{~kJ} / \mathrm{mol}$, while IL-6 protein system showed stability with both drugs with values
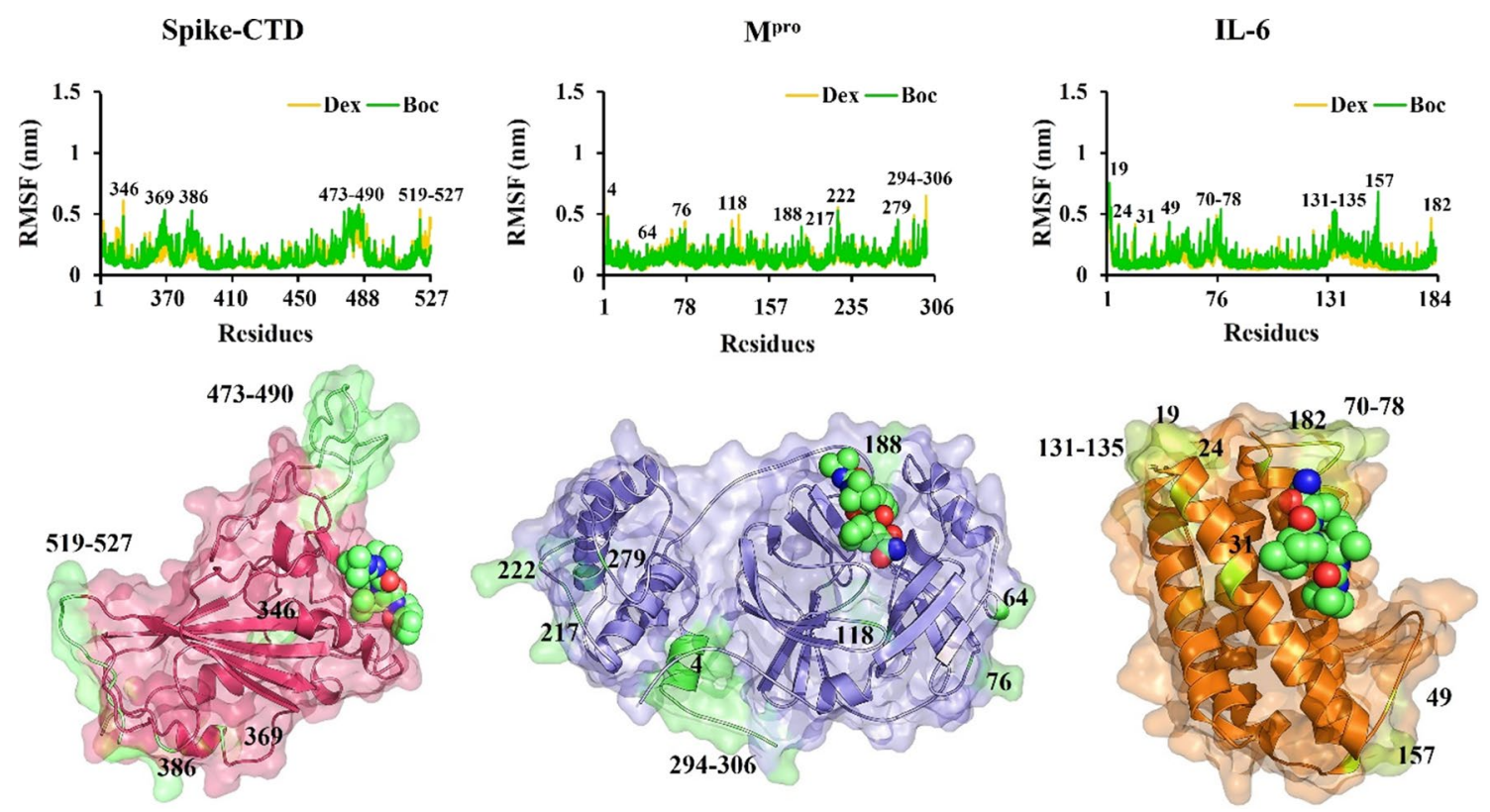

Fig. 5 RMSF plots of all three proteins with the two drugs from onemicrosecond simulations per system. Only proteins-Boc complexes images are presented under the plots. Proteins are shown in both surface and cartoon representation, and the drug is shown in sphere representation, color-coded by element ( $\mathrm{N}$ : blue, $\mathrm{O}$ : red, $\mathrm{H}$ : white), and only carbon atoms are green. The residues of relatively high fluctuations in the proteins' structures are highlighted in green in all the images and marked on the plot 


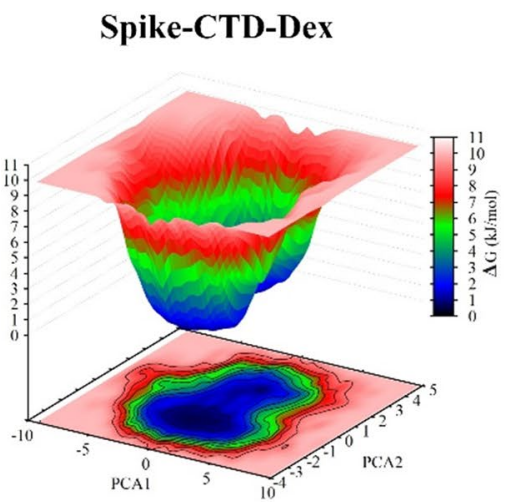

Spike-CTD-Boc

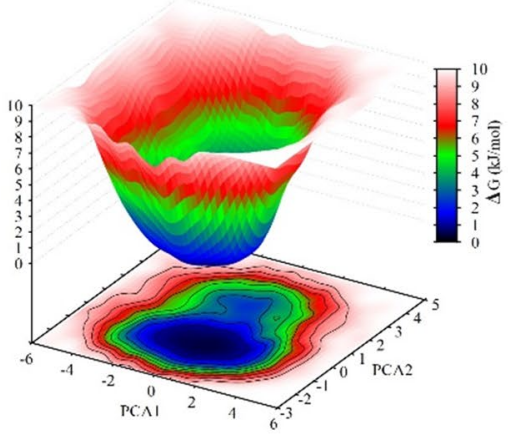

M $^{\text {pro-Dex }}$
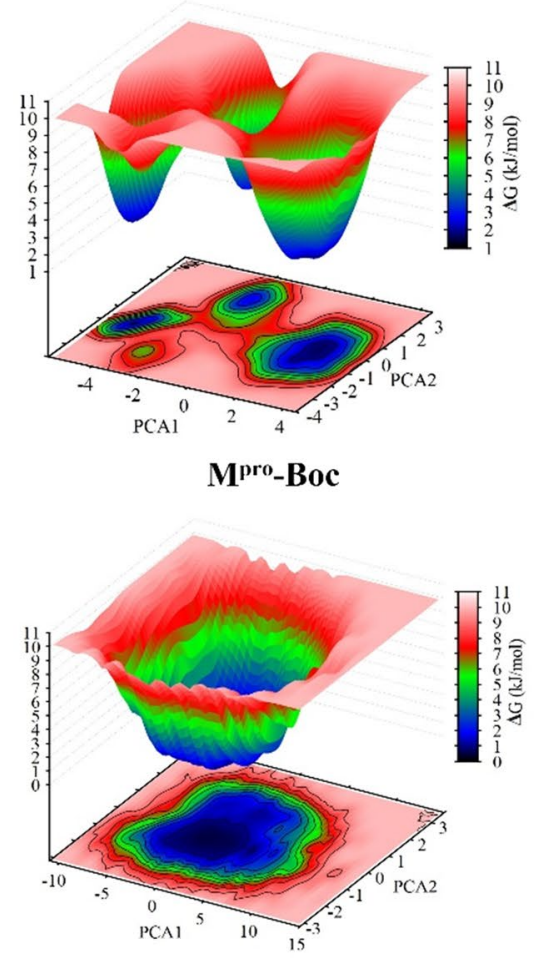

IL-6-Dex

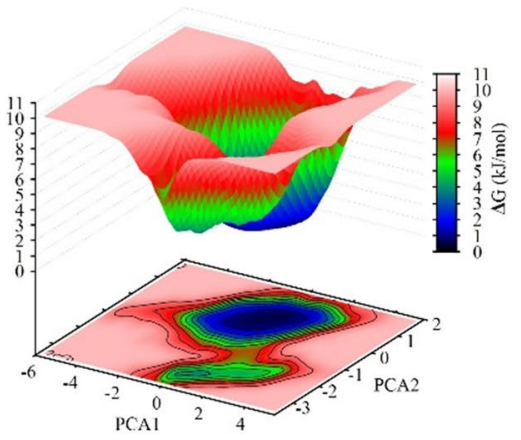

IL-6-Boc

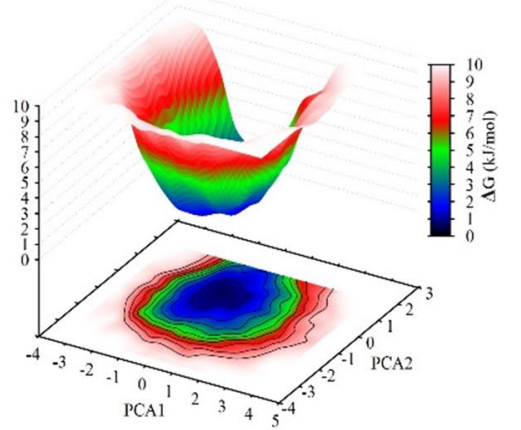

Fig. 6 3D Gibbs free energy landscapes (FEL) of each of the protein-drug systems obtained during one-microsecond MD simulations

of 9.97 (Il-6-Dex) and $9.90 \mathrm{~kJ} / \mathrm{mol}$ (IL-6-Boc). $\mathrm{M}^{\text {pro }}$ protein complexed with Dex exhibited better stability compared to the Boc drug-protein system. The outcomes from the FEL analysis further validated the results from other analyses that all six protein-drug systems were stable. However, for $\mathrm{M}^{\text {pro }}$ with Dex two energy minima were observed showing two possible binding modes (mode 1: Fig. 7. B, mode 2: Fig. S6), and both binding modes exhibited several interactions between protein and drug as depicted in the images.

\section{Binding mode analysis of targeted proteins with drugs}

Clustering was performed to analyze the variations in the different poses of bound drugs at each protein-binding site. Exploration of the binding modes of Dex and Boc drugs at the binding pocket of all three proteins via cluster analysis showed one significant cluster, thus validating the output from other analyses that the binding mode of drugs was very stable and no major variation in the interactions between drugs and proteins. The center of the cluster was used as a representative structure for all protein-drug complexes (Fig. 7).

H-bonding analysis was performed to compute the number of hydrogen bonds between the drugs and proteins (Fig. 8). A representative complex structure of
spike-CTD-Dex showed that Dex formed various h-bonding contacts with the residues of spike-CTD including R403, Y453, S494, G496, and Y505 (Shi et al. 2020; Wang et al. 2020). Dex drug also made hydrophobic contacts with V483. Analysis of the binding mode of spike-CTD and hACE2 complex crystal structure exhibited that Y453, G496, and Y505 were the key residues that were located at the interface between spike-CTD and hACE2, thus forming h-bonding and hydrophobic interactions with hACE2 (Wang et al. 2020).

These results indicated that Dex was able to bind at the binding interface of spike-CTD, which typically interacted with hACE2 very well via several interaction modes such as hydrogen and hydrophobic contacts. Spike-CTD-Boc complex analysis showed that the Boc drug was also able to interact with the binding interface of spike-CTD via h-bonding and hydrophobic contacts.

Exploration of the binding mode of Dex and Boc drugs with $\mathrm{M}^{\text {pro }}$ indicated that they formed strong intermolecular binding interactions with the key active site residues of $\mathrm{M}^{\text {pro }}$ including T45, S46, M165, E166, L167, and Q189 (Shamsi et al. 2020; Mohammad et al. 2020). These contacts between the drugs and proteins were formed via several strong h-bonding $(2.03 \AA$, $2.26 \AA, 2.71 \AA$ ) and hydrophobic interactions $(4.0 \AA)$. Comparison of the binding modes that revealed from this study with crystal-bound structure of Boc 
Fig. 7 Binding mode interactions of protein-drug complexes from one-microsecond simulations per system. A spike-CTD-Dex, B Mpro-Dex, C IL-6-Dex, D spike-CTDBoc, E Mpro-Boc, F IL-6-Boc. Proteins are shown in ribbon presentation and colored in pink (spike-CTD), light blue ( $\mathrm{M}^{\mathrm{pro}}$ ), and orange (IL-6). Interacting residues are shown in stick representation. Dex and Boc drugs are colored by element (N: blue, $\mathrm{O}$ : red, $\mathrm{H}$ : white), and only carbon atoms are colored in yellow and green, respectively. Hydrogen bonding and hydrophobic interactions are indicated in dashed lines colored green and purple, respectively. Nonpolar hydrogens are hidden for clarity
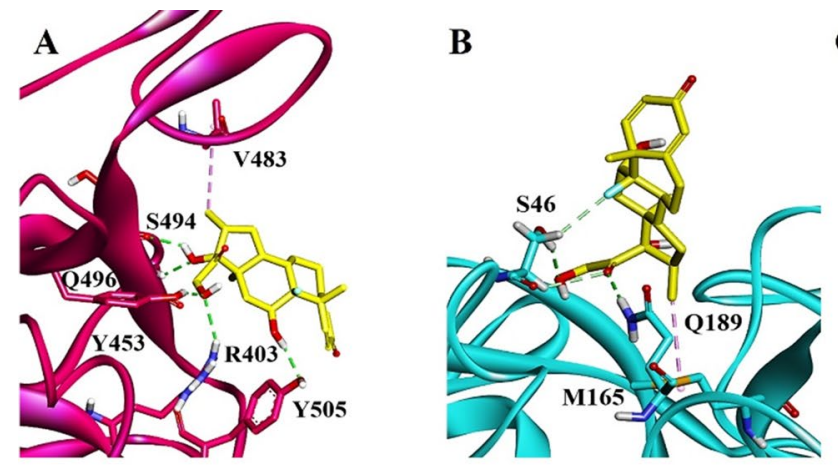

C
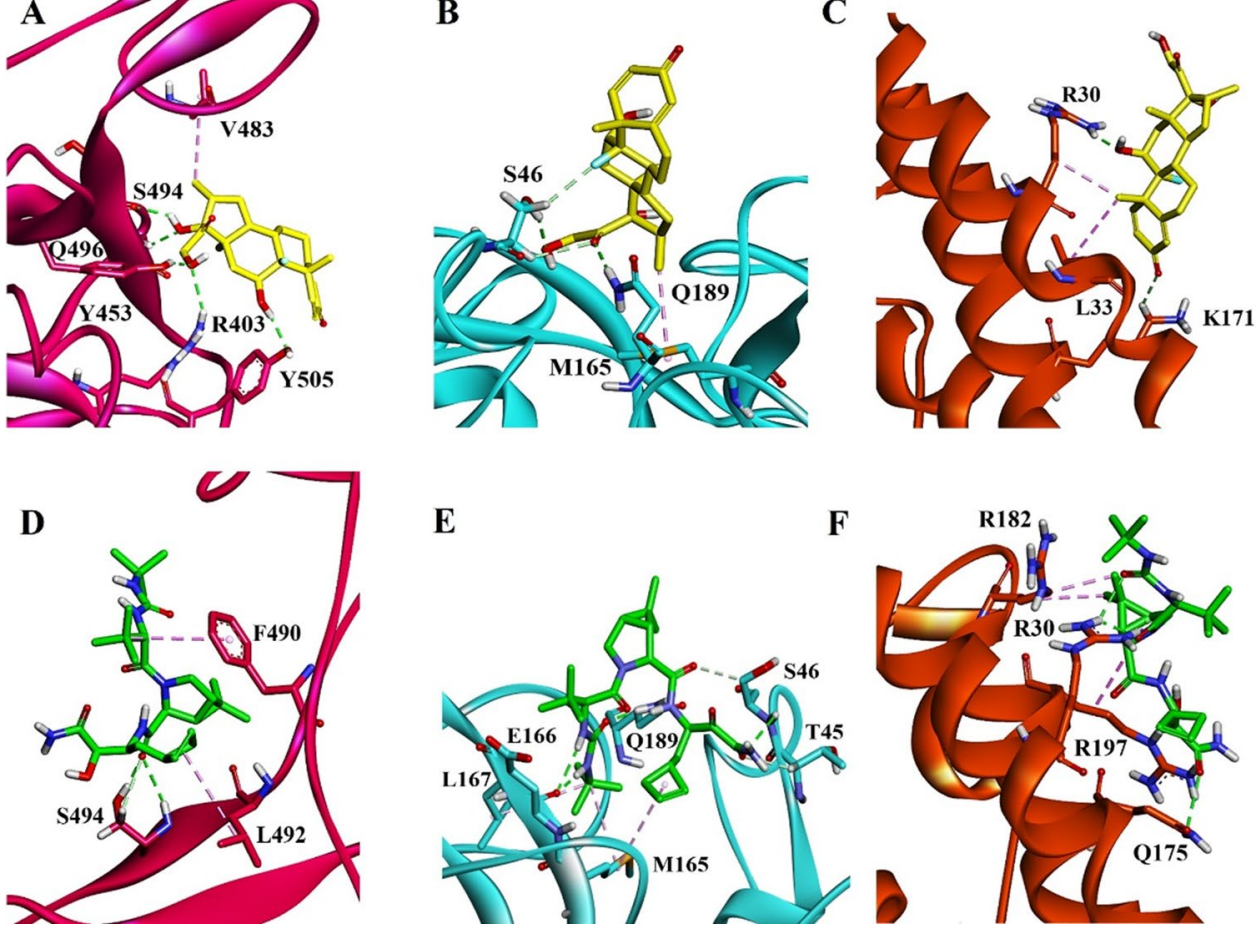

F

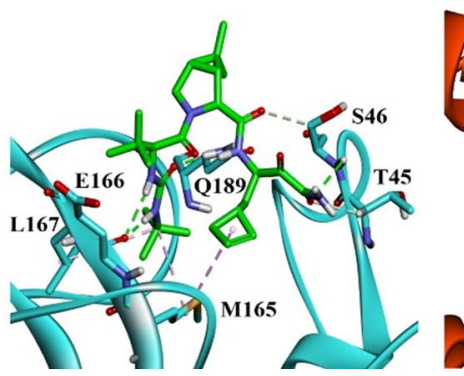

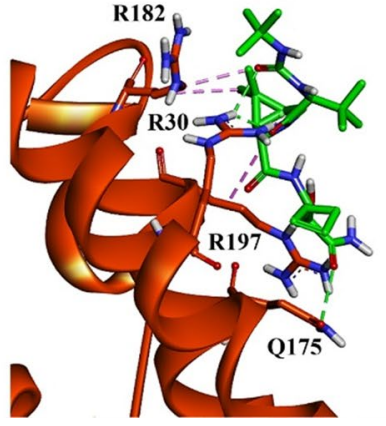

Spike-CTD

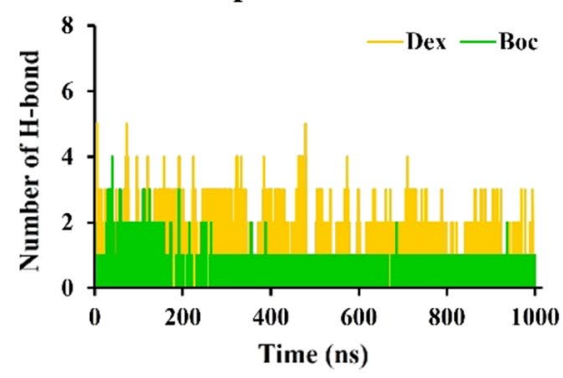

Mpro

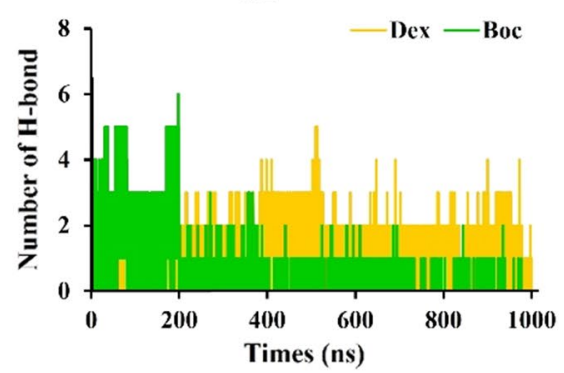

IL-6

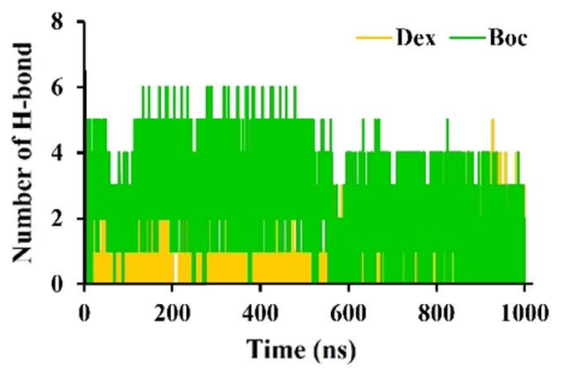

Fig. 8 Number of hydrogen bonds in each of the protein-drug systems from one-microsecond simulations per system

with $\mathrm{M}^{\text {pro }}$ showed the presence of several common interactions between the predicted binding modes and crystal structure complexes. These contacts confirmed the importance of these residues in the effective binding of the ligands at the active site of $\mathrm{M}^{\text {pro }}$. IL-6-Dex and IL-6-Boc complexes were also analyzed, and both drugs were able to make strong contacts with the interacting residues of R30, L33, K171, Q175, R182, and R197.

\section{Steered molecular dynamics and umbrella sampling}

SMD trajectories provided the initial configurations for US windows which were equally spaced in $0.02 \mathrm{~nm}$ windows, yielding a total of 90 windows of $10 \mathrm{~ns}$ each. The free energy of binding (PMF) is the difference between the minima where the drug is bound to the protein and the plateau value when binding mode contacts between drug and protein were effectively absent (Fig. 9).

The umbrella sampling histograms of each molecule had presented excellent overlaps. The free energies of drug binding to the three proteins obtained via the US are listed in Table 1 . The highest magnitude $\left(\Delta \mathrm{G}=-28.02 \mathrm{~kJ} \mathrm{~mol}^{-1}\right)$ of binding free energy was obtained for spike-CTD protein. Moreover, both drugs Dex and Boc also showed good binding affinity with other two proteins as well.

\section{Effect of drug binding on spike-CTD interactions with hACE2}

Other than exploring the binding modes and stability of both drugs at the three key targets of COVID-19, another key objective of the current study was to investigate the effect of 


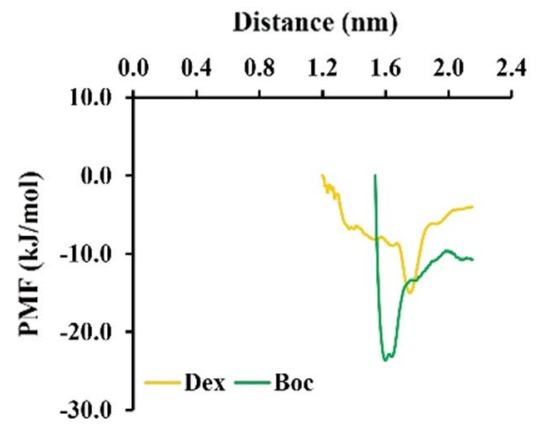

Spike-CTD

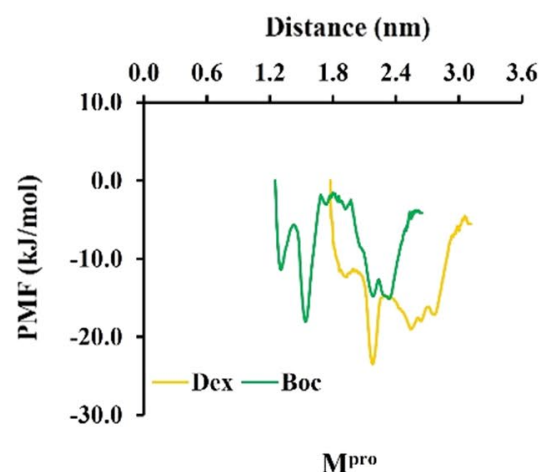

$\mathbf{M}^{\text {pro }}$

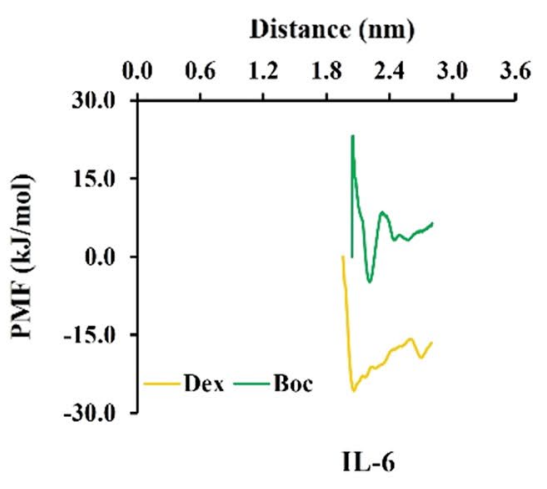

Fig. 9 Potential of mean force obtained from umbrella sampling for Dex and Boc drugs binding to the spike-CTD, M ${ }^{\text {pro }}$, and IL-6 proteins. The minimum distance represents the drug-bound state, and the maximum distance represents the drug unbound state with the protein

Table 1 Binding affinity $(\mathrm{kJ} / \mathrm{mol})$ of protein-drug complexes extracted from the PMF values

\begin{tabular}{llll}
\hline & Spike & M $^{\text {pro }}$ & IL-6 \\
\hline Dex & -25.72 & -23.45 & -17.93 \\
Boc & -28.02 & -18.20 & -23.64 \\
\hline
\end{tabular}

the drugs binding at the spike-CTD ability to interact with hACE2. To answer this query, the binding conformations of both drugs with spike-CTD were retrieved from one- $\mu$ s simulations and were subjected to another simulation in the presence of hACE2. RMSD analysis showed that both viral and human proteins were stable throughout the simulations in both complex systems containing the drugs (Fig. S5).

Further analysis of these simulations including h-bonding examination at the interface of spike-CTD and hACE2 revealed that hACE2 was not able to show optimal binding with the interface of spike-CTD in the presence of the drugs (Fig. 10).

Receptor hACE2 showed a few interactions with the interface in spike-CTD complexed with Dex and Boc drugs at the very early stages of simulation to $6 \mathrm{~ns}$ and $11 \mathrm{~ns}$, respectively, as shown in the h-bonding graph (Fig. 10). However, hACE2 was not able to maintain these few interactions and could not continue binding at the specified

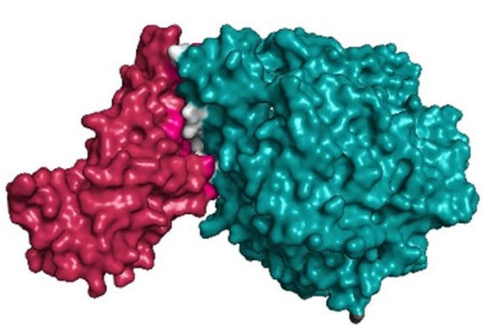

No drug

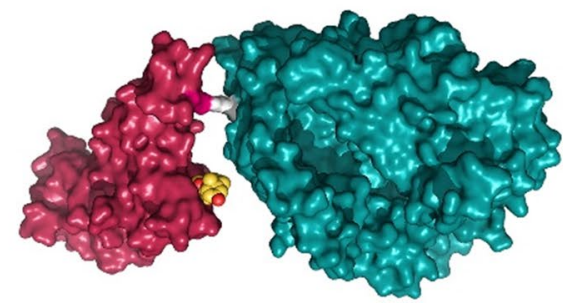

$6 \mathrm{~ns}$

Presence of Dexamethasone

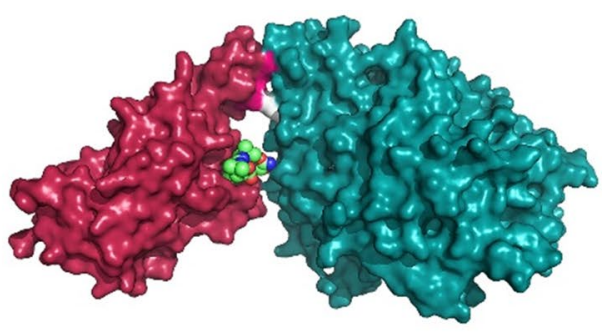

$11 \mathrm{~ns}$

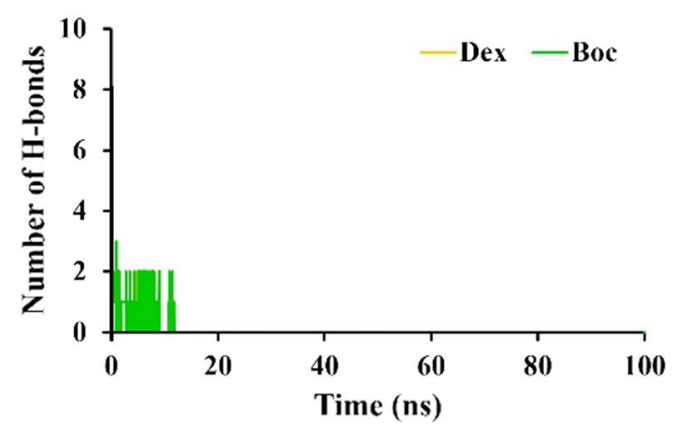

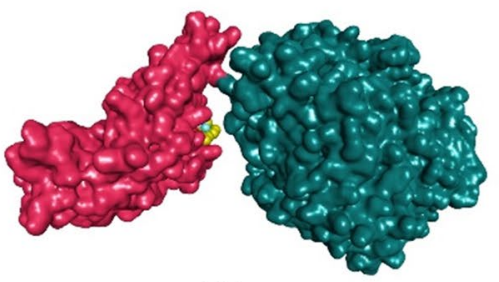

$100 \mathrm{~ns}$

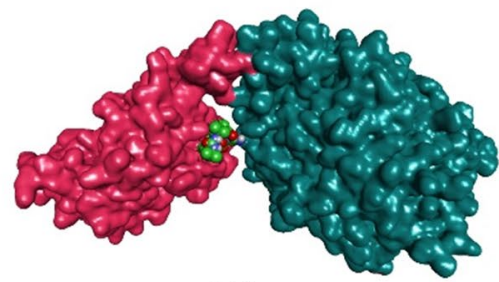

$100 \mathrm{~ns}$

Fig. 10 Binding mode interactions of protein-protein in the presence of the drugs. Both proteins are shown in surface and ribbon presentations with spike-CTD and hACE2 colored in red and green, respectively. Bound drugs with spike-CTD protein are displayed as spheres 
interface of spike-CTD. The presence of the drugs at the binding interfaces did not allow hACE2 to have putative bindings as was observed in the crystal structure complexes of spike-CTD with hACE2 with numerous interactions.

\section{Conclusions}

We explored the binding configurations of Dex and Boc drugs against vital COVID-19 protein targets including viral spike-CTD, $\mathrm{M}^{\text {pro }}$, and IL-6 using microsecond-long simulations and enhanced sampling methods. Overall analyses depicted the stability of drug-bound protein complexes. Lower RMSD values for the proteins and drugs at the binding cavity of the three proteins showed that protein-drug complexes did not undergo much deviation during the simulations. The radius of gyration analysis indicated the compactness of all simulated systems. The proteins exhibited that binding pockets did not show much flexibility in the presence of the drugs, and the residues located in these binding regions were less flexible. Relatively higher flexible regions were located at $\mathrm{N}$ - and $\mathrm{C}$-termini of the proteins along with the coil regions. Clustering of the simulated conformations further confirmed the presence of single-dominated binding modes for both drugs in the binding pocket of the complexed proteins.

Detailed analysis of the binding conformations of both drugs indicated their ability to interact with the binding site residues through $\mathrm{h}$-bonding and hydrophobic interactions. The free energy landscape retrieved from PCA showed the existence of the lowest energy conformations of protein-drug complexes. PMF graphs which were built from SMD and US sampling methods indicated the strong binding of drugs with all three proteins. Comparatively, both Dex and Boc drugs exhibited better binding with spike-CTD than the other two protein targets with binding free energy values of -25.72 and $-28.02 \mathrm{~kJ} / \mathrm{mol}$, respectively. $\mathrm{M}^{\text {pro }}$-Dex complex showed better binding and stability than $\mathrm{M}^{\text {pro }}-\mathrm{Boc}$, while for IL-6 protein, Boc binding was relatively better than Dex. Outcomes from the simulations of viral spikeCTD complexed with Dox and Boc drugs in the presence of hACE2 revealed that the binding of the drugs at the interface of spike-CTD caused the reduction of the interaction for hACE2 which finally lead to no interactions with the human receptor at the spike-CTD interface. Overall, the findings from this study revealed the optimal binding modes of both drugs with all three key targets of COVID-19 including spike-CTD which prevented its interaction at the interface with the ACE2 human receptor.

Supplementary Information The online version contains supplementary material available at https://doi.org/10.1007/s11696-021-01843-0.
Acknowledgements MA acknowledges the SEED research grant (1902144083) from the University of Sharjah. The authors acknowledge the use of the HPC Facility at the University of Sharjah for part of the work reported in this paper. AAM acknowledges the University of Sharjah support of SEED grant (VC-GRC-SR-83-2015), competitive grants (160-2142-029-P and 150-2142-017-P).

\section{Declarations}

Conflict of interest The authors declare that they do not have any conflict of interest.

Human and animal rights This research does not involve human participants and/or animals.

Informed consent There is no need for informed consent.

\section{References}

Abraham MJ, Murtola T, Schulz R, Páll S, Smith JC, Hess B, Lindah E (2015) Gromacs: High performance molecular simulations through multi-level parallelism from laptops to supercomputers. SoftwareX 1:19-25

Arooj M, Shehadi I, Nassab CN, Mohamed AA (2020) Physicochemical stability study of protein-benzoic acid complexes using molecular dynamics simulations. Amino Acids 52(9):1353-1362

Bentel JM, Thomas MA, Rodgers JJ, Arooj M, Gray E, Allcock R, Fermoyle S, Mancera RL, Cannell P, Parry J (2017) ErdheimChester disease associated with a novel, complex BRAF p Thr599Val600delinsArgGlu mutation. BMJ Case Reports. https://doi.org/ 10.1136/bcr-2017-219720

Castagnoli R, Votto M, Licari A, Brambilla I, Bruno R, Perlini S, Rovida F, Baldanti F, Marseglia GL (2020) Severe acute respiratory syndrome coronavirus 2 (SARS-CoV-2) infection in children and adolescents: a systematic review. JAMA Pediatr 174(9):882-889

Chandrasekharan P, Vento M, Trevisanuto D, Partridge E, Underwood MA, Wiedeman J, Katheria A, Lakshminrusimha S (2020) Neonatal resuscitation and postresuscitation care of infants born to mothers with suspected or confirmed SARS-CoV-2 infection. Am J Perinatol 37(8):813-824

Ciliberto G, Cardone L (2020) Boosting the arsenal against COVID19 through computational drug repurposing. Drug Discov Today 25(6):946-948

Darden T, York D, Pedersen L (1993) Particle mesh Ewald: An N. $\log (\mathrm{N})$ method for Ewald sums in large systems. J Chem Phys 98(12):10089-10092

Della-Torre E, Campochiaro C, Cavalli G, De Luca G, Ciceri F, Zangrillo A, Dagna L (2020) Targeting IL-1, IL-6 or GM-CSF in COVID-19 Response to: 'More evidences on which biologic and which pathway is key in severe-critical COVID-19 pneumonia' by Ferraccioli. Ann Rheum Dis. https://doi.org/10.1136/annrh eumdis-2020-218612

Dodda LS, Cabeza de Vaca I, Tirado-Rives J, Jorgensen WL (2017) LigParGen web server: an automatic OPLS-AA parameter generator for organic ligands. Nucleic Acids Res 45:W331-W336. https://doi.org/10.1093/nar/gkx312

Elfiky A (2020) Ribavirin, Remdesivir, Sofosbuvir, Galidesivir, and Tenofovir against SARS-CoV-2 RNA dependent RNA polymerase (RdRp): A molecular docking study. Life Sci 253:117592

Fu L, Ye F, Feng Y, Yu F, Wang Q, Wu Y, Zhao C, Sun H, Huang B, Niu P (2020) Both Boceprevir and GC376 efficaciously inhibit 
SARS-CoV-2 by targeting its main protease. Nat Commun 11:4417

Gavor E, Choong YK, Er SY, Sivaraman H, Sivaraman J (2020) Structural basis of SARS-CoV-2 and SARS-CoV-antibody interactions. Trens Immunol 41(11):1006-1022

Goodsell DS, Morris GM, Olson A (1996) Automated docking of flexible ligands: applications of AutoDock. J Mol Recognit 9(1):1-5

Gurwitz D (2020) Angiotensin receptor blockers as tentative SARSCoV-2 therapeutics. Drug Dev Res 81:537-540

Harb LH, Arooj M, Vrielink A, Mancera R (2017) Computational site-directed mutagenesis studies of the role of the hydrophobic triad on substrate binding in cholesterol oxidase. Proteins 85(9):1645-1655

Hasan A, Paray BA, Hussain A, Qadir FA, Attar F, Aziz FM, Sharifi M, Derakhshankhah H, Rasti B, Mehrabi M et al (2020) A review on the cleavage priming of the spike protein on coronavirus by angiotensin-converting enzyme-2 and furin. J Biomol Struct Dyn 39(8):3025-3033

Ho L-J, Luo S-F, Lai J (2015) Biological effects of interleukin-6: clinical applications in autoimmune diseases and cancers. Biochem Pharmacol 97(1):16-26

http://zarbi.chem.yale.edu/ligpargen/

https://www.strodel.info/index-files/lecture/generateFES.py

Iqbal Z, Morahan G, Arooj M, Sobolev AN, Hameed S (2019) Synthesis of new arylsulfonylspiroimidazolidine-2, 4-diones and study of their effect on stimulation of insulin release from MIN6 cell line, inhibition of human aldose reductase, sorbitol accumulations in various tissues and oxidative stress. Eur J Med Chem 168:154-175

Jackson LA, Anderson EJ, Rouphael NG, Roberts PC, Makhene M, Coler RN, McCullough MP, Chappell JD, Denison MR, Stevens L et al (2020) An mRNA vaccine against SARS-CoV-2-preliminary report. N Engl J Med 383:1920-1931

Jani V, Koulgi S, Uppuladinne VN, Sonavane U, Joshi R (2021) An insight into the inhibitory mechanism of phytochemicals and FDA-approved drugs on the ACE2-Spike complex of SARSCoV-2 using computational methods. Chem Pap Do. https://doi. org/10.1007/s11696-021-01680-1

Johnson RM, Vinetz JM (2020) Dexamethasone in the management of covid-19. BMJ. https://doi.org/10.1136/bmj.m2648

Khan SU, Htar TT (2020) Deciphering the binding mechanism of Dexamethasone against SARS-CoV-2 Main Protease: Computational molecular modelling approach. Doi: https://doi.org/10. 26434/chemrxiv.12517535.

Ma C, Sacco MD, Hurst B, Townsend JA, Hu Y, Szeto T, Zhang X, Tarbet B, Marty MT, Chen Y et al (2020) Boceprevir, GC-376, and calpain inhibitors II, XII inhibit SARS-CoV-2 viral replication by targeting the viral main protease. Cell Res 30(8):678-692

Mehta P, Porter JC, Manson JJ, Isaacs JD, Openshaw PJ, McInnes IB, Summers C, Chambers R (2020) Therapeutic blockade of granulocyte macrophage colony-stimulating factor in COVID19-associated hyperinflammation: challenges and opportunities. Lancet Respir Med 8:822-830

Mohammad T, Shamsi A, Anwar S, Umair M, Hussain A, Rehman MT, AlAjmi MF, Islam A, Hassan M (2020) Identification of high-affinity inhibitors of SARS-CoV-2 main protease: Towards the development of effective COVID-19 therapy. Virus Res 288:198102

Nayeem SM, Sohail EM, Ridhima G, Reddy MS (2021) Target SARSCoV-2: Computation of Binding energies with drugs of Dexamethasone/Umifenovir by Molecular Dynamics using OPLS-AA force field. Res Biomed Eng 1-10.

Ngo ST, Vu KB, Bui LM, Vu VV (2019) Effective estimation of ligandbinding affinity using biased sampling method. ACS Omega 4:3887-3893

Rahman MM, Saha T, Islam KJ, Suman RH, Biswas S, Rahat EU, Hossen MR, Islam R, Hossain MN, Mamun A (2020) Virtual screening, molecular dynamics and structure-activity relationship studies to identify potent approved drugs for Covid-19 treatment. J Biomol Struct Dyn. https://doi.org/10.1080/07391102.2020. 1794974

Shamsi A, Mohammad T, Anwar S, AlAjmi MF, Hussain A, Rehman M, Islam A, Hassan M (2020) Glecaprevir and Maraviroc are highaffinity inhibitors of SARS-CoV-2 main protease: possible implication in COVID-19 therapy. Biosci Rep 40(6):BSR20201256

Shehadi IA, Rashdan HR, Abdelmonsef A (2020) Homology Modeling and Virtual Screening Studies of Antigen MLAA-42 Protein: Identification of Novel Drug Candidates against Leukemia-An In Silico Approach. Comp Math Meth Med. https://doi.org/10. 1155/2020/8196147

Shi R, Shan C, Duan X, Chen Z, Liu P, Song J, Song T, Bi X, Han $\mathrm{C}, \mathrm{Wu} \mathrm{L}$ et al (2020) A human neutralizing antibody targets the receptor binding site of SARS-CoV-2. Nature 584(7819):120-124

Somers W, Stahl M, Seehra JS (1997) $1.9 \AA$ crystal structure of interleukin 6: implications for a novel mode of receptor dimerization and signaling. EMBO J 16(5):989-997

Sud S (2020) COVID-19 and keeping clean: a narrative review to ascertain the efficacy of personal protective equipment to safeguard healthcare workers against SARS-CoV-2. Hosp Pediatr 10(7):570-576

Trott O, Olson AJ (2009) AutoDock Vina: Improving the speed and accuracy of docking with a new scoring function, efficient optimization, and multithreading. J Comput Chem 31:455-461

Ullrich S, Nitsche C (2020) The SARS-CoV-2 main protease as drug target. Bioorg Med Chem Lett 30(17):127377

Wang S-Q, Du Q-S, Zhao K, Li A-X, Wei D-Q, Chou K-C (2007) Virtual screening for finding natural inhibitor against cathepsin-L for SARS therapy. Amino Acids 33(1):129-135

Wang Q, Zhang Y, Wu L, Niu S, Song C, Zhang Z, Lu G, Qiao C, $\mathrm{Hu}$ Y, Yuen K-Y et al (2020) Structural and functional basis of SARS-CoV-2 entry by using human ACE2. Cell 181(4):894-904

Wei D-Q, Zhang R, Du Q-S, Gao W-N, Li Y, Gao H, Wang S-Q, Zhang X, Li A-X, Sirois S (2006) Anti-SARS drug screening by molecular docking. Amino Acids 31(1):73-80

Publisher's Note Springer Nature remains neutral with regard to jurisdictional claims in published maps and institutional affiliations. 